\title{
REGIONS OF MEROMORPHY DETERMINED BY THE DEGREE OF BEST RATIONAL APPROXIMATION ${ }^{1}$
}

\author{
E. B. $S A F F^{2}$
}

ABSTRACT. In this paper we investigate the relationship between the degree of best rational approximation to a given function $f(z)$ and the regions in which $f(z)$ is meromorphic. We show, for example, that if rational functions $r_{n}(z)$ of respective types $(n, \nu)$, i.e., rational functions with $\nu$ free poles, converge geometrically (as $n \rightarrow \infty$ ) to $f(z)$ on a closed Jordan region $E$, then $f(z)$ must be meromorphic in a region which contains $E$ in its interior.

1. Introduction. 'The theory which relates the regions of analyticity of a given function to the degree of convergence of polynomials of best approximation to that function is well known (see $[3, \S 4.7]$ ). The corresponding theory for rational approximation, however, is not yet complete. In the present paper we develop this theory by showing that the degree of convergence of rational functions of best approximation to a given function can be used to describe regions in which the approximated function is meromorphic.

To be more explicit, let $E$ be the closed interior of a Jordan curve in the $z$-plane and let $w=\phi(z)$ map the complement of $E$ conformally onto the exterior of the unit circle in the w-plane so that $\phi(\infty)=\infty$ and $\phi^{\prime}(\infty)>0$. Let $\Gamma_{\sigma}(\sigma>1)$ denote generically the locus $|\phi(z)|=\sigma$ and let $E_{\sigma}$ be the interior of $\Gamma_{\sigma}$.

We shall approximate functions defined on the set $E$ by rational functions of prescribed types. A rational function $r_{n v}(z)$ is said to be of type $(n, \nu)$ if it is of the form

$$
r_{n \nu}(z)=p_{n}(z) / q_{\nu}(z), \quad q_{\nu}(z) \not \equiv 0,
$$

where $p_{n}(z)$ is a polynomial of degree at most $n$ and $q_{\nu}(z)$ is a polynomial of degree at most $\nu$. The following result concerning the degree of approximation by rational functions of type $(n, \nu)$ is due to $\mathrm{J}$. L. Walsh [4, p. 2]:

Received by the editors June 29, 1970.

AMS 1969 subject classifications. Primary 3070, 4117.

Key words and phrases. Regions of meromorphy, best rational approximation, closed Jordan region, rational function of type $(n, \nu)$, polynomial of least squares approximation, Faber polynomials.

1 This paper is dedicated to the author's first child, Lisa Jill Saff, on whose day of birth, April 24, 1970, the main result of this paper was proved.

2 The research of the author was supported, in part, by NSF grant GF-19275. 
THEOREM 1. Suppose $f(z)$ is a function which is analytic on the set $E$ and meromorphic with at most $\nu$ poles (multiplicity included) in the region $E_{\rho}(1<\rho \leqq \infty)$. Then there exists a sequence of rational functions $r_{n v}(z)$ of respective types $(n, \nu)$ which satisfy

$$
\underset{n \rightarrow \infty}{\lim \sup }\left[\max \left|f(z)-r_{n v}(z)\right| ; z \text { on } E\right]^{1 / n} \leqq 1 / \rho \quad(<1) .
$$

The object of this paper is to establish the converse to Theorem 1, namely

THEOREM 2. Let $f(z)$ be a function continuous on the closed bounded Jordan region $E$. If rational functions $r_{n \nu}(z)$ of respective types $(n, \nu)$, $\nu$ fixed, $n=0,1, \cdots$, exist satisfying (1), then the function $f(z)$ can be extended so as to be analytic on $E$ and meromorphic with at most $\nu$ poles in the region $E_{\rho}$.

For the special case of approximation by polynomials, i.e., for $\nu=0$, Theorem 2 was proved by Walsh [3, p. 78] who extended an earlier result due to $\mathrm{S}$. Bernstein. (In fact, for $\nu=0$ Theorem 2 holds not only for $E$ a closed Jordan region but for any compact point set $E$ whose complement is connected and regular.) The validity of Theorem 2 for $\nu>0$ is mentioned by Walsh in $[4$, p. 6] as an open problem. The difficulty in extending the proof of the theorem from $\nu=0$ to $\nu>0$ lies in the fact that for $\nu>0$ the finite poles of the $r_{n \nu}(z)$, whose locations are not specified, may be everywhere dense in the complement of $E$. In $\S 2$ we shall prove Theorem 2 by applying Hadamard's classical results on the location of polar singularities.

As an immediate consequence of Theorems 1 and 2 we obtain the following result concerning best rational approximation:

Theorem 3. Suppose $f(z)$ is analytic on the closed bounded Jordan region $E$ and for each pair $(n, \nu)$ let

$$
E_{n v}(f) \equiv \inf \left\{\left[\max \left|f(z)-R_{n \nu}(z)\right| ; z \text { on } E\right]\right\},
$$

where the infimum is taken over all rational functions $R_{n \nu}(z)$ of type $(n, \nu)$. Let

$$
1 / \rho_{\nu} \equiv \limsup _{n \rightarrow \infty} E_{n \nu}(f)^{1 / n}, \quad \nu=0,1, \cdots
$$

Then:

(i) The sequence $\rho_{\nu}$ is nondecreasing.

(ii) $\rho_{\nu}$ is the largest number $\sigma$ with the property that $f(z)$ is meromorphic with at most $\nu$ poles in the region $E_{\sigma}$.

(iii) If $\rho_{\nu}>\rho_{\nu-1}$, then $f(z)$ has precisely $\nu$ poles in $E_{\rho_{\nu}}$. 
(iv) $\rho \equiv \lim _{\nu \rightarrow \infty} \rho$, is the largest number $\sigma$ with the property that $f(z)$ is meromorphic in $E_{\sigma}$.

(v) $f(z)$ has infinitely many poles in $E_{\rho}$ if and only if $\rho_{\nu}<\rho$ for $\nu=0,1, \cdots$.

The proof of Theorem 3 is left to the reader.

2. Proof of Theorem 2. The proof is based upon the following result due to Hadamard [1, pp. 24-37]:

THEOREM 4. Let the function $g(z)=\sum_{0}^{\infty} a_{n} z^{n}$ be analytic at $z=0$ and for each pair $(n, \nu)$ put

$$
D_{n \nu} \equiv \operatorname{det}\left[\begin{array}{llll}
a_{n} & a_{n+1} & \cdots & a_{n+\nu} \\
a_{n+1} & a_{n+2} & \cdots & a_{n+\nu+1} \\
\cdot & \vdots & & \vdots \\
\cdot & \cdot & & \cdot \\
a_{n+\nu} & a_{n+\nu+1} & \cdots & a_{n+2 \nu}
\end{array}\right] .
$$

Let $l_{\nu} \equiv \lim \sup _{n \rightarrow \infty}\left|D_{n \nu}\right| 1 / n, \nu=0,1, \cdots$, and set $l_{-1} \equiv 1$. Then the function $g(z)$ is meromorphic with at most $\nu$ poles in the disk $|z|<l_{\nu-1} / l_{\nu}$.

If in Theorem 4 some $l_{i}$ is zero, then we interpret the quotient $l_{\nu-1} / l_{\nu}$ as $+\infty$ for every $\nu \geqq m$, where $m$ is the smallest subscript for which $l_{i}=0$.

We shall first prove Theorem 2 for the case where $E$ is a closed disk. To do, we need the following result concerning the minimum value of a certain function of $\nu$ complex variables:

Lemma 1. Let $C^{\nu}$ denote the set of all ordered $\nu$-tuples $Z=\left(z_{1}, z_{2}, \cdots, z_{v}\right)$ of complex numbers and for $k=1,2, \cdots, \nu+1$ let

$$
L_{k}(Z)=b_{k}+\sum_{i=1}^{\infty} b_{k i} z_{i}
$$

Let $B^{(\mu)}, 1 \leqq \mu \leqq \nu+1$, be the matrix obtained by deleting the $\mu$ th row from the $(\nu+1)-b y-\nu$ matrix

$$
\left[\begin{array}{llll}
b_{11} & b_{12} & \cdots & b_{1 v} \\
b_{21} & b_{22} & \cdots & b_{2 v} \\
\vdots & \vdots & \vdots \\
\dot{b}_{v+1,1} & \dot{b}_{v+1,2} & \cdots & \dot{b}_{v+1, v}
\end{array}\right]
$$

Suppose that the set $I \equiv\left\{\mu \mid \operatorname{det} B^{(\mu)} \neq 0\right\}$ is nonempty and for each $\mu \in I$ let $Z^{(\mu)}$ be the unique solution of the system of $\nu$ linear equations $L_{k}(Z)=0$, $1 \leqq k \leqq \nu+1, k \neq \mu$. 
Then the function

$$
L(Z) \equiv \sum_{k=1}^{p+1}\left|L_{k}(Z)\right|, \quad Z \in C^{\nu}
$$

attains its absolute minimum at some point $Z^{(\mu)}, \mu \in I$.

Proof. We suppose, as we may, that $I=\{m, m+1, \cdots, \nu+1\}$, $1 \leqq m \leqq \nu+1$, so that $\operatorname{det} B^{(\mu)}=0$ for $1 \leqq \mu<m$. If for some $\mu, m \leqq \mu$ $<\nu+1$, we have $Z^{(\mu)}=Z^{(\nu+1)}$, then

$$
L_{\mu}\left(Z^{(\mu)}\right)=L_{\mu}\left(Z^{(\nu+1)}\right)=0,
$$

and so $L\left(Z^{(\mu)}\right)=0$, which obviously implies the lemma. Hence we assume that $Z^{(\mu)} \neq Z^{(\nu+1)}$ for $m \leqq \mu<\nu+1$.

Let $W_{i}$, for $1 \leqq i<m$, be a nonzero vector in the null space of the matrix $B^{(i)}$ and for $m \leqq i \leqq \nu$ put $W_{i}=Z^{(i)}-Z^{(\nu+1)}$. We shall show that the $\nu$ vectors $W_{i}$ are linearly independent. Suppose to the contrary that for some $\mu, 1<\mu \leqq \nu$, we have

$$
W_{\mu}=\sum_{i=1}^{\mu-1} c_{i} W_{i}
$$

It is easy to verify that

$$
L_{k}\left(W_{i}\right)-b_{k}=0, \quad \text { for } 1 \leqq i, k \leqq \nu, \quad k \neq i,
$$

and so from (2) it follows that $L_{\mu}\left(W_{\mu}\right)-b_{\mu}=0$. Thus $L_{k}\left(W_{\mu}\right)-b_{k}=0$ for $1 \leqq k \leqq \nu$, i.e. the nonzero vector $W_{\mu}$ belongs to the null space of the matrix $B^{(v+1)}$. But this is impossible since $\operatorname{det} B^{(v+1)} \neq 0$. Hence the $W_{i}$ form a basis for $C^{\nu}$.

Each vector $Z$ in $C^{\nu}$ can thus be written in the form

$$
Z=Z^{(v+1)}+\sum_{i=1}^{\nu} t_{i} W_{i}
$$

for suitable constants $t_{i}$. Since

$$
L_{k}\left(W_{k}\right)-b_{k}=L_{k}\left(Z^{(k)}\right), \quad \text { for } m \leqq k \leqq \nu,
$$

and

$$
\begin{aligned}
L_{v+1}\left(W_{k}\right)-b_{v+1} & =0, & & \text { for } 1 \leqq k<m, \\
& =-L_{\nu+1}\left(Z^{(v+1)}\right), & & \text { for } m \leqq k \leqq \nu,
\end{aligned}
$$

we deduce from (3) and (4) that 


$$
\begin{aligned}
L_{k}(Z) & =t_{k} L_{k}\left(Z^{(k)}\right), & & \text { for } m \leqq k \leqq \nu, \\
& =\left(1-\sum_{k=m}^{\nu} t_{k}\right) L_{\nu+1}\left(Z^{(v+1)}\right), & & \text { for } k=\nu+1 .
\end{aligned}
$$

Now let $\left|L_{q}\left(Z^{(q)}\right)\right|=\min \left\{\left|L_{k}\left(Z^{(k)}\right)\right|, m \leqq k \leqq \nu+1\right\}$. Then

$$
\begin{aligned}
L(Z) & \geqq \sum_{k=m}^{\nu+1}\left|L_{k}(Z)\right|=\sum_{k=m}^{\nu}\left|t_{k} L_{k}\left(Z^{(k)}\right)\right|+\left|\left(1-\sum_{k=m}^{\nu} t_{k}\right) L_{\nu+1}\left(Z^{(v+1)}\right)\right| \\
& \geqq\left|L_{q}\left(Z^{(q)}\right)\right|\left(\sum_{k=m}^{\nu}\left|t_{k}\right|+\left|1-\sum_{k=m}^{\nu} t_{k}\right|\right) \\
& \geqq\left|L_{q}\left(Z^{(q)}\right)\right|=L\left(Z^{(q)}\right),
\end{aligned}
$$

which proves the lemma.

Using Cramer's rule it is easy to show that for $\mu \in I$ we have

$$
\left|L_{\mu}\left(Z^{(\mu)}\right)\right|=|\operatorname{det} B| /\left|\operatorname{det} B^{(\mu)}\right|,
$$

where

$$
B \equiv\left[\begin{array}{lllll}
b_{1} & b_{11} & b_{12} & \cdots & b_{1 v} \\
b_{2} & b_{21} & b_{22} & \cdots & b_{2 v} \\
\vdots & \vdots & \vdots & \vdots \\
\dot{b}_{v+1} & \dot{b}_{v+1,1} & \dot{b}_{v+1,2} & \cdots & b_{v+1, v}
\end{array}\right]
$$

Hence Lemma 1 asserts that

$$
L(Z) \geqq|\operatorname{det} B| / \max _{1 \leqq \mu \leqq \nu+1}\left|\operatorname{det} B^{(\mu)}\right|, \quad Z \in C^{\nu} .
$$

We can now prove

Lemma 2. Let $g(z)=\sum_{0}^{\infty} a_{n} z^{n}, D_{n v}$, and $l_{\nu}$ be defined as in Theorem 4 and let $\Delta_{\sigma}$ denote the closed disk $|z| \leqq \sigma$. If there exists a sequence of rational functions $s_{n \nu}(z)$ of respective types $(n, \nu), \nu$ fixed $, n=0,1, \cdots$, which satisfy

(6) $\lim \sup \left[\max \left|g(z)-s_{n \nu}(z)\right| ; z \text { on } \Delta_{\sigma}\right]^{1 / n} \leqq 1 / \rho \quad(1<\rho \leqq \infty)$,

then we have

$$
l_{\nu-1} / l_{\nu} \geqq \rho \sigma .
$$

Proof. Since the poles of the $s_{n \nu}(z)$ lie in $|z|>\sigma$, we may write $s_{n \nu}(z)=p_{n}(z) / q_{n}(z)$, where $p_{n}(z)$ is a polynomial of degree at most $n$ and $q_{n}(z)$ is a polynomial of the form 


$$
q_{n}(z)=\prod_{k=1}^{\prime}\left(1-\alpha_{n k} z\right)=1+\sum_{k=1}^{\prime} c_{n k} z^{k}
$$

where $\left|\alpha_{n k}\right|<1 / \sigma$ for $k=1, \cdots, \nu$. Note that $\left|q_{n}(z)\right| \leqq 2^{\text {v for }}|z|=\sigma$ and hence

$$
\begin{aligned}
{\left[\max \left|g(z)-s_{n v}(z)\right|\right.} & \left.; z \text { on } \Delta_{\sigma}\right]^{2} \\
& \geqq(2 \pi \sigma)^{-1} \int_{|z|=\sigma}\left|g(z)-s_{n \nu}(z)\right|^{2}|d z| \\
& \geqq(2 \pi \sigma)^{-1} 2^{-2 \nu} \int_{|z|=\sigma}\left|g(z) q_{n}(z)-p_{n}(z)\right|^{2}|d z| .
\end{aligned}
$$

Since the $n$th section of the Taylor development

$$
g(z) q_{n}(z)=\sum_{k=0}^{\infty}\left(a_{k}+\sum_{i=1}^{\nu} a_{k-i} c_{n i}\right) z^{k}
$$

is the polynomial of degree at most $n$ of least squares approximation to the function $g(z) q_{n}(z)$ on $|z|=\sigma$, we have for $n \geqq \nu$ the inequalities

$$
\begin{aligned}
\int_{|z|=\sigma}\left|g(z) q_{n}(z)-p_{n}(z)\right|^{2}|d z| & \geqq \int_{|z|=\sigma}\left|\sum_{k=n+1}^{\infty}\left(a_{k}+\sum_{i=1}^{\nu} a_{k-i} c_{n i}\right) z^{k}\right|^{2}|d z| \\
& \geqq 2 \pi \sigma \sum_{k=n+1}^{n+\nu+1}\left|a_{k}+\sum_{i=1}^{\nu} a_{k-i} c_{n i}\right|^{2} \sigma^{2 k} \\
& \geqq \frac{2 \pi \sigma}{\nu+1}\left[\sum_{k=n+1}^{n+\nu+1}\left|a_{k}+\sum_{i=1}^{\nu} a_{k-i} c_{n i}\right| \sigma^{k}\right]^{2}
\end{aligned}
$$

the last inequality follows from the Cauchy-Schwarz inequality.

Now let $D_{n, \nu-1}^{(\mu)}$ denote the determinant of the matrix obtained by deleting the $\mu$ th row from the matrix

$$
\left[\begin{array}{llll}
a_{n} & a_{n-1} & \cdots & a_{n+1-\nu} \\
a_{n+1} & a_{n} & \cdots & a_{n+2-\nu} \\
\vdots & \vdots & & \vdots \\
a_{n+\nu} & a_{n+\nu-1} & \cdots & a_{n+1}
\end{array}\right] .
$$

Then from (5) we deduce that

$$
\sum_{k=n+1}^{n+\nu+1}\left|a_{k}+\sum_{i=1}^{\nu} a_{k-i} c_{n i}\right| \geqq\left|D_{n+1-\nu, \nu}\right| / \max _{1 \leqq \mu \leqq \nu+1}\left|D_{n, \nu-1}^{(\mu)}\right|,
$$


provided the denominator of the right-hand side is nonzero. Assuming this to be the case we combine (8) and (9) to obtain

$$
\left[\max \left|g(z)-s_{n \nu}(z)\right| ; z \text { on } \Delta_{\sigma}\right] \geqq A \sigma^{n}\left|D_{n+1-\nu, \nu}\right| / \max _{1 \leqq \mu \leqq+1}\left|D_{n, \nu-1}^{(\mu)}\right| \text {, }
$$

where $A$ is a positive constant independent of $n$. But note that if $D_{n, \nu-1}^{(\mu)}=0$ for every $\mu, 1 \leqq \mu \leqq \nu+1$, then we have $D_{n+1-\nu, \nu}=0$, and so the inequality

$$
\max _{1 \leqq \mu \leqq \nu+1}\left|D_{n, \nu-1}^{(\mu)}\right|\left[\max \left|g(z)-s_{n \nu}(z)\right| ; z \text { on } \Delta_{\sigma}\right] \geqq A \sigma^{n}\left|D_{n+1-\nu, \nu}\right|
$$

holds without any restrictions on the $D_{n, \nu-1}^{(\mu)}$. In his proof of Theorem 4 Hadamard shows that

$$
\limsup _{n \rightarrow \infty}\left|D_{n, \nu-1}^{(\mu)}\right|^{1 / n} \leqq l_{\nu-1}, \quad 1 \leqq \mu \leqq \nu+1 .
$$

Hence from (6) and (10) there follows $l_{\nu-1} / \rho \geqq \sigma l_{\nu}$, and the last inequality obviously implies (7), even for the case $\rho=\infty$.

From Theorem 4 and Lemma 2 we deduce that Theorem 2 holds for $E=\Delta_{\sigma}$. We consider another special case of Theorem 2 in

Lemma 3. Theorem 2 is valid if $E$ is bounded by an analytic Jordan curve.

Proof. Let $\phi(z)$ be defined as in $\S 1$ and let $\tau \equiv 1 / \phi^{\prime}(\infty)$. The function $w=\Phi(z) \equiv \tau \phi(z)$ maps the complement of $E$ conformally onto $|w|>\tau$ and satisfies $\Phi(\infty)=\infty, \Phi^{\prime}(\infty)=1$. Let $\psi(w)$ be the inverse of $\Phi(z)$ and note that since the boundary of $E$ is an analytic Jordan curve there exists an $r, 0<r<\tau$, so that $\psi(w)$ is analytic and univalent in $|w| \geqq r, w \neq \infty$.

It is clear from (1) that the function $f(z)$ is analytic in the interior $D$ of $E$. Hence if $F_{n}(z)$ denotes the Faber polynomial of degree $n$ for $E$, the expansion

$$
f(z)=\sum_{0}^{\infty} a_{n} F_{n}(z), \quad a_{n}=\frac{1}{2 \pi i} \int_{|w|=\tau} \frac{f(\psi(w))}{w^{n+1}} d w,
$$

converges to $f(z)$ in $D$ (see $[2, \S 14]$ ). Since $\left|a_{n}\right| \leqq A / \tau^{n}$, it follows from $[2$, p. 107] that the function

$$
h(w) \equiv \sum_{0}^{\infty} a_{n}\left(F_{n}(\psi(w))-w^{n}\right)
$$

is analytic for $|w| \geqq r$ and vanishes at $\infty$. From (11) we have

$$
f(\psi(w))=g(w)+h(w), \quad r \leqq|w| \leqq \tau,
$$


where $g(w) \equiv \sum_{0}^{\infty} a_{n} w^{n}$ is analytic in $|w|<\tau$ and continuous on $|w| \leqq \tau$. To prove Lemma 3 it thus suffices to show that the function $g(w)$ is meromorphic with at most $\nu$ poles in the disk $|w|<\rho \tau$.

For this purpose we first note that

$$
\frac{1}{2 \pi i} \int_{|t|=\tau} \frac{r_{n \nu}(\psi(t))}{t-w} d t=s_{n \nu}(w), \quad|w|<\tau
$$

where $s_{n \nu}(w)$ is a rational function of type $(n, \nu)$. We briefly justify (13). Suppose $r_{n \nu}(z)$ has a pole of order $m$ at the point $\psi\left(w_{0}\right),\left|w_{0}\right|>\tau$, with singular part $S(z) \equiv \sum_{1}^{m} B_{k} /\left(z-\psi\left(w_{0}\right)\right)^{k}$. For each positive integer $k$ the function

$$
\theta(t) \equiv\left(t-w_{0}\right)^{k} /\left(\psi(t)-\psi\left(w_{0}\right)\right)^{k}(t-w), \quad|w|<\tau,
$$

is analytic for $|t| \geqq \tau$ and is zero for $t=\infty$. Hence from Cauchy's integral formula we have

$$
\frac{1}{2 \pi i} \int_{|t|=\tau} \frac{d t}{\left(\psi(t)-\psi\left(w_{0}\right)\right)^{k}(t-w)}=\frac{1}{2 \pi i} \int_{|t|=\tau} \frac{\theta(t) d t}{\left(t-w_{0}\right)^{k}}=\frac{-\theta^{(k-1)}\left(w_{0}\right)}{(k-1) !} .
$$

It is easy to verify that $\theta^{(k-1)}\left(w_{0}\right)$ is of the form

$$
\theta^{(k-1)}\left(w_{0}\right)=\sum_{j=1}^{k} C_{j} /\left(w-w_{0}\right)^{j}
$$

and so

$$
\frac{1}{2 \pi i} \int_{|t|=\tau} \frac{S(\psi(t))}{t-w} d t=\sum_{j=1}^{m} B_{j}^{*} /\left(w-w_{0}\right)^{j}, \quad|w|<\tau .
$$

From (14) and the fact that the function

$$
P_{k}(w) \equiv \frac{1}{2 \pi i} \int_{|t|=\tau} \frac{\psi(t)^{k}}{t-w} d t, \quad|w|<\tau
$$

is a polynomial of degree $k$ in $w$, we obtain (13).

Now since

$$
\frac{1}{2 \pi i} \int_{|t|=\tau} \frac{h(t)}{t-w} d t=0, \quad|w|<\tau .
$$

it follows from (12) and (13) that

$$
g(w)-s_{n \nu}(w)=\frac{1}{2 \pi i} \int_{|t|=\tau} \frac{f(\psi(t))-r_{n \nu}(\psi(t))}{t-w} d t, \quad|w|<\tau,
$$


and hence inequality (1) implies that

$$
\limsup _{n \rightarrow \infty}\left[\max \left|g(w)-s_{n \nu}(w)\right| ;|w| \leqq \sigma\right]^{1 / n} \leqq 1 / \rho, \quad \sigma<\tau .
$$

Thus as a consequence of Lemma 2 we have $l_{\nu-1} / l_{\nu} \geqq \rho \sigma$, and on letting $\sigma \rightarrow \tau$ we obtain $l_{\nu-1} / l_{\nu} \geqq \rho \tau$. It now follows from Theorem 4 that $g(w)$ is meromorphic with at most $\nu$ poles in $|w|<\rho \tau$. This proves Lemma 3.

From Lemma 3 it is easy to deduce Theorem 2. For if $E$ is a closed bounded Jordan region and $1<R<\rho$, then there exists [3, \$2.1] a set $E^{\prime} C E$ such that $E^{\prime}$ is the closed interior of an analytic Jordan curve and such that $E \subset E_{R}^{\prime}$. Since inequality (1) holds with $E$ replaced by $E^{\prime}$, Lemma 3 implies that $f(z)$ is meromorphic with at most $\nu$ poles in the region $E_{\rho}^{\prime}$. But $E_{\rho / R} \subset\left(E_{R}^{\prime}\right)_{\rho / R}=E_{\rho}^{\prime}$. Therefore, since $R(>1)$ is arbitrary, it follows that $f(z)$ is meromorphic with at most $\nu$ poles in $E_{\rho}$. This completes the proof of Theorem 2.

\section{REFERENCES}

1. J. Hadamard, "Essai sur l'étude des fonctions données par leur développement de Taylor," in Oeuvres de Jacques Hadamard. Vol. 1, Centre National de la Recherche Scientifique, Paris, 1968. MR 37 \#6158.

2. A. I. Markuševič, Theory of functions of a complex variable. Vol. 3, GITTL, Moscow, 1950; English transl., Prentice-Hall, Englewood Cliffs, N. J., 1967. MR 12, 87; MR $35 \# 6799$.

3. J. L. Walsh, Interpolation and approximation by rational functions in the complex domain, 3rd ed., Amer. Math. Soc. Colloq. Publ., vol. 20, Amer. Math. Soc., Providence, R. I., 1960. MR 36 \#1672a.

4. - The convergence of sequences of rational functions of best approximation with some free poles, Proc. Sympos. Approximation of Functions (General Motors Res. Lab., 1964), Elsevier, Amsterdam, 1965, pp. 1-16. MR 32 \#4441.

UNIVERSITY OF SOUTh FLORIDA, TAMPA, FLORIDA 33620 